\title{
New look video in vocational education: What factors contribute to its success?*
}

\author{
Dr Justus H. Lewis \\ Ms Vivienne Blanksby \\ Royal Melbourne Institute of Technology
}

\begin{abstract}
As knowledge expands exponentially, so does the need for industry to keep pace with new information, techniques and theories. Video learning has the potential to contribute speedily and effectively to meeting this need. What factors should be taken into account to ensure the success of video learning? Recent developments at RMIT and in the UK are used as illustrative examples.
\end{abstract}

During the eighties, government interest in the potential for education and training to bring about significant improvements in workforce competence and hence promote economic development has been a major phenomenon.

This has been particularly noticeable in the UK, where the establishment of the Open Tech Program in 1983 was an attempt to address the many reasons why working adults are unable or unwilling to participate in education. The Program aimed to bring about significant changes in accessibility, relevance and motivation in vocational training as provided by both the formal education system and the training resources of companies (See Tolley, 1983 and Tinsley, 1985). Via the Open Tech, the Manpower Services Commission provided forty-five million pounds as pump-priming finance to education and industry training bodies for new programs using Open Learning methods.

When the Open Tech had run its course in 1987, the evidence suggested that while some programs had been dramatically successful, many had

* This paper was presented at the EdTech'88 Conference in September 1988 [http:/ / ascilite.org.au / aset-archives/confs / edtech88/edtech88_contents.html], but was not available in time to be included with the Conference papers. 
foundered on an over-emphasis on the production of expensive learning resources and inadequate attention to the environmental aspects of learning - an imbalance which the Open College, successor to the Open Tech, is attempting to rectify.

In Australia steps to achieve a comparable level of development of industrial education and liaison have started later. The last two years have seen significant moves in this direction in terms of discussion of the issues. As yet, however, no comparable injection of government funding has taken place and the emphasis has been on the need for educational institutions to extend their funding base to meet the changing situation.

This paper has a particular focus on the uses of video in vocational education. Educational television has been with us in Australia for thirty years, but the almost universal availability of VCR equipment has dramatically changed its potential as an educational tool within the space of approximately five years. The ephemeral qualities of broadcast television and the cumbersome nature of film projection equipment have always limited the possibilities of using these media as integrated components of educational programs. The portability, reviewability, and accessibility of video make possible a completely new range of approaches to the use of the visual medium.

It is important to realise that in terms of learning the viewing of video material may be as fruitless as conventional teaching. This paper then is concerned to identify the factors which provide for optimum use of the advantages of the medium. We argue that video-led education can provide a very successful means of training and higher order learning in the work place if an approach is adopted which integrates the educational content of the learning material with a concept of management of the learning environment.

In recent months, several departments in RMIT have been experimenting and evaluating different educational uses of video. Several overseas institutions have been contacted and visited. This paper draws on these experiences to illustrate its main points.

\section{Why video?}

It may be helpful to review the strengths of video. As suggested above, video playback equipment is now widely available at reasonable cost; modern technology has made scanning of tapes quicker and easier; and there are portable cameras with automatic focussing, light adjustment and basic editing facilities which make it possible for novice users to obtain usable footage with minimum training and instruction.

Human beings have the potential to be endlessly interesting to each other. Hence a medium which captures body language and sound qualities has 
greater intrinsic appeal than the printed word or the computer monitor. There is an increasing need for the rapid dissemination of 'state-of-the- art' information. There is also a natural human curiosity about the people who are the sources of this information. How many stories have we heard from students who have been attracted to or repelled from certain subjects because of the performance of the teacher in that subject? 'A picture is worth a thousand words'. Frequently, the demonstration of how to carry out a particular process or the modelling of certain types of behaviour conveys more to the learner than an hour of verbal explanation. Video allows both to be integrated in a medium which can be easily controlled by the learner.

\section{Promoting interactive viewing}

Criticisms most frequently levelled at the use of video and television in education are that they are essentially passive media, and that material presented this way will be quickly forgotten. This is certainly true if it is used as it was frequently in the past as a fill-in, vaguely intended to enrich but not seen as a real part of the course by the students.

Tony Bates (1988) of the Open University has distinguished the three main educational characteristics of television (by which he means all forms of televisual presentation) as the delivery, presentational and control characteristics.

Delivery characteristics enable the distribution of learning stimuli beyond the point of origin (as with the book or radio) and presentational characteristics can provide rich vicarious experiences which cannot be achieved in other ways without enormous expense and inconvenience.

It is, however, the control characteristic of the video cassette that sets it apart from other versions of the medium. Control enables learners not only to view programs at convenient times but to achieve more effective learning than in many other situations.

The program can be stopped at any convenient point for reflection, discussion or practice of what has just been seen before moving on to further material. If necessary the same scene can be watched several times to ensure that all the finer points are grasped. However these features will be ignored by many learners unless the encouragement to do so is clear.

Open University research shows that when learners are viewing alone they are unlikely to stop the tape before the end of a program (Durbridge 1986, quoted in Bates 1988). If a video program is designed as a self-study resource, it is important to build in design features to encourage viewers to stop the tape. Bates identifies the following six: 
1. Use of segments

2. Clear stopping points

3. Use of activities

4. Indexing

5. Close integration with other media (e.g. text. discussion)

6. Concentration on audio-visual aspects

Follow up activities to the viewing of the program are critical to its usefulness and, therefore, challenge the designer of the study program to provide a framework for interactivity. Video material can and should be supplemented by fill-in response sheets, workbooks, assignments and related practical activities. The way in which these are presented will depend on the current state of knowledge and confidence expected of the audience and will at times require simple recall of facts and at others more challenging analysis and interpretation. A fill-in sheet may also serve as a memory jogger if the learner is then required to practise a skill demonstrated by the video program.

Ideally, however, learners will not be watching alone. The value of discussion of the material presented should be emphasised. Although the use of video material, as with any other open learning resource, can provide the basis of a study unit in situations where there is no expert teacher, the viewing of such material in groups even as small as two provides great enhancement to the learners' participation in the experience. The group situation provides both a motivational impetus to allocate time to thinking about the issues presented and a sounding board for comparing ideas and checking the legitimacy of responses.

\section{The importance of immediate response}

Our current RMIT involvement with video-led education includes a number of formats and a variety of target groups - the use of video to introduce and reinforce the teaching of practical skills (the laying of plasterboard for building industry employees), complex interpersonal skills (as in interviewing and counselling situations), presentation of current medical procedures (undergraduate and continuing education of medical laboratory scientists, nurses and doctors) and the understanding of abstract concepts (continuing education for practising engineers).

In all of these situations, we have found that the possibility of students responding immediately to what they have participated in vicariously by viewing the video adds another dimension to their learning environment which, in fact, would not necessarily be present in the original situation if they were participants in that situation. To achieve this, however, requires thought and pre-planning from the managers of the project. 
In practical skills training, the chance to practise the required skill should be as close as possible to the demonstration and will, therefore, require forethought to enable sensible sequencing, the availability of appropriate equipment and a suitable setting.

Interpersonal technique development should if possible include opportunities for analysis, discussion and role play immediately following the presentation of case study material. These can be guided by carefully designed print support material to enable an inexpert facilitator to lead a group.

Where in depth analysis of such skills is required, the opportunities provided by recording and immediate playback of students' performance in role play situations enable rich exploration and reflection on aspects of interpersonal performance.

When dealing with abstract concepts, the opportunity for immediate discussion and review of complex lecture material as enabled by the technique of Tutored Video Instruction has been shown to add significant value to the learning experience

\section{Tutored video instruction (TVI) as a problem-based methodology}

TVI is a low cost method in which small, tutor-led groups view unedited video recordings of live lectures. It has been used successfully by Stanford University (Stanford Instructional Television Network, International Program in Engineering Studies) for a number of years to teach a variety of post-graduate courses to engineers in their work environment. A similar centre has been set up at Aston University in the UK (the Centre for Extension Education). The Centre has offered a number of the Aston courses to industry and is currently embarking on an MBA program. At the Heriot Watt University of Technology in Edinburgh, Video Services International has operated for a number of years. This provides a facility to its associate members which enables them to access courses currently available in video format and the evaluations of these courses which have been made by previous users. It also commissions the preparation of new courses in TVI format. A slightly different approach is being taken by Strathclyde University which has recently produced a video-led series of programs on Expert Systems which it is intended should be used in the context of a TVI-type program.

Recent TVI and TVI-type developments at RMIT include the presentation of part of the Master in Systems Engineering course in TVI mode and the development of a video and workbooks on the treatment of chronic transfusion patients by the Department of Applied Biology. 
TVI is a good example of an active use of video in the classroom. The active involvement of the students has a number of features:

- the tape is stopped every 5 to 10 minutes by either the tutor or the students for class discussion;

- students are encouraged to find their own solutions to problems;

- students are encouraged to relate what is presented on the tape to their own work situations.

In addition, it has been found (Stephen, 1986) that TVI used in industry has produced peripheral benefits to companies because the horizontal and vertical groupings of students have provided opportunities for fostering cooperation and communication amongst staff. This agrees with earlier findings at Stanford.

\section{Implementing TVI}

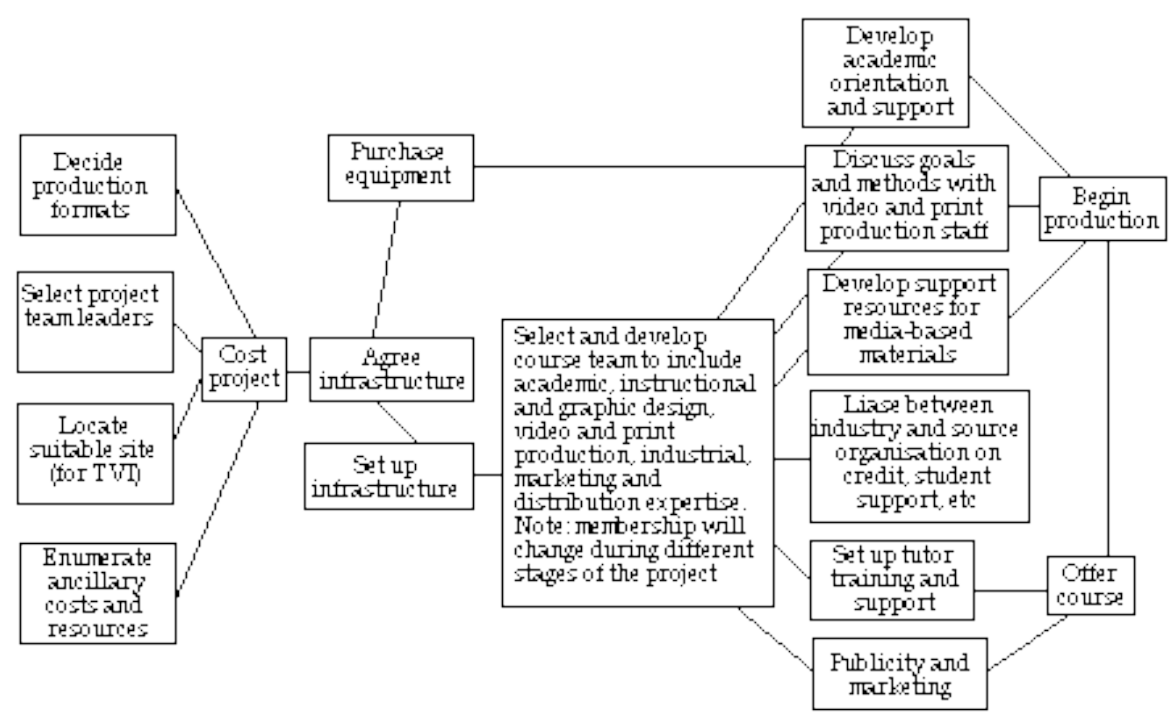

Figure 1: Draft Project Chart for the production of TVI and Open Learning courses

What are the factors that facilitate the successful implementation of TVI? The management of the learning environment needs to be carefully considered as there are a range of factors to be taken into account which differ significantly both from what is required for individual teachers to operate in 'normal' classrooms and from the successful management of more traditional distance education. 
Initial extensive cross-sectional collaboration is required. In the RMIT MSE project, for example, members of the development team included the course coordinator, a technical media adviser and an educational development consultant. Sustained input from all over a period of time resulted in the support of a senior section Head in obtaining a suitable room and equipment to begin the first trials.

A further factor is adequate tutor support for students. This will be dealt with in more detail below.

Another is the provision of support for the lecturers who will make the TVI presentation. Although one of the advantages of the TVI mode is that the lecturer operates in a close to normal classroom environment, it is still reassuring to the lecturer to have access to advice on methods of presentation that will be more appropriate in the TVI format and, in particular, advice on the preparation of overheads and other illustrative material. This also enhances the standard of presentation. The need for this pre-production liaison and input must be recognised and allowed for. Another related aspect is the group dynamics of the classroom. Part of the effectiveness of the final video presented to the TVI students is dependent on them feeling that they have had access to the original interactive classroom environment. The necessity to video the original classroom may be perceived as restrictive by the students. Hence the need for a special TVI classroom which will be as 'user friendly' as possible for both lecturers and students.

There are other practical details to be considered. How should the class hand- out material be prepared for both classes? What are the mechanisms by which it can be speedily duplicated and distributed?

A further practical detail is the liaison between the course advisers and the industry or industries which are the potential market for the courses. What training should be given to tutors and what channels of communication provided between them and the originators of the course material?

A graphic representation of some of these considerations is shown in Figure 1.

\section{The role of the tutor}

The role of the tutor has already been referred to as one of the important factors in the successful implementation of TVI. One of the more interesting features of the tutor's role is that the tutor is not a subject expert, although in at least some subject areas it is desirable that tutors should have a basic grasp of and interest in the area which is being studied. The more important characteristics of successful tutors are their abilities to play a leadership role in the areas described below. 
The tutor operates in a number of areas:

- the tutor is a catalyst for the group discussion;

- the tutor acts as liaison between the lecturers and the TVI students;

- the tutor has an important role in feedback and evaluation.

\section{The tutor as a catalyst}

The tutor must be someone who is respected by the students. He or she is the key link in determining whether the TVI group will operate in a problem solving mode and whether this will be student generated and application oriented. Students prefer tutors who will draw them into discussion rather than answer their questions directly.

\section{The tutor as liaison person}

The tutor has an important liaison role between the TVI students and the lecturer in charge of the subject. For this reason, it is important that tutors should be adequately briefed. In many cases it may be more appropriate and cost-effective if the tutor is supplied by the company or organisation using the TVI.

\section{The tutor in feedback and evaluation}

The tutor's input to the source institution in providing local information regarding the specific needs of the industry, the characteristics of the students and their reactions to the TVI material play a vital role in the fine tuning of the program.

\section{An integrated management approach}

The characteristics of the video medium enable the provision, under controlled conditions, of experiences otherwise unavailable to learners whether it be the tireless demonstration of particular technical skills, the opportunity to analyse vividly presented interpersonal situations or the chance to hear and discuss state of the art developments in technology as presented by a distant expert.

Much of the success of the use of this medium depends on how well the environmental conditions for the provision of a video-led program are managed.

Of significant importance to the context of the training or education program is the broad issue of the attitude of management personnel and their willingness to provide the resources both tangible - tutors, equipment, learning materials - and more abstract - time during working hours, places suitably equipped for study and high profile encouragement. 
Good study resources can fail to make an effective study program if there is no adequate commitment from the sponsoring organisation. The necessary support elements of the program should be fully understood and accepted. Advice on the provision of support arrangements is a vital part of the total delivery package from the source institution and will need to form an integral part of program planning.

Selection of appropriate program leaders is another important factor. Even in individualised Open Learning situations, learners will benefit from the support of a work place based tutor, mentor or supervisor. Where possible, however, arrangements for group activities are highly desirable. The choice of leader, who need not be a subject specialist in many cases, requires care. Such a person will often be the critical influence in the marketing of the program to the students - stimulating enthusiasm and enabling them to see how the program works.

An infrastructure to handle the distribution of materials, the recording of student progress, liaison and feedback to the source institution and other related matters will minimise the trivial problems which can unnecessarily create negative feelings about a program.

With due attention to questions such as these, video-led learning can be a very positive experience for individual learners and their sponsoring organisations.

\section{References}

Bates, Tony. (1988). Television, learning and distance education. ICDE Bulletin, 16, January, 29-38.

Gibbons, J. F. (1987). Tutored videotape instruction: an approach to educational productivity. The Stanford Engineer, Spring/Summer, 11-19.

Stephen, K. D. (1986). The use of distance learning in industry. Proceedings of the Institution of Mechanical Engineers, 200(No B2), 131-137.

Tinsley, David. (1985). The Open Tech Programme: a progress report. Media in Education and Development, March, 2-5.

Tolley, George. (1983). The Open Tech Programme: A new initiative for adults. International Journal of Lifelong Education, 2(4), 327-340.

Please cite as: Lewis, J. H. and Blanksby, V. (1988). New look video in vocational education: What factors contribute to its success? Australian Journal of Educational Technology, 4(2), 109-117. http:/ / www.ascilite.org.au/ajet/ajet4/.html 